\title{
Research Article \\ Variance Bound of ACF Estimation of One Block of fGn with LRD
}

\author{
Ming $\mathrm{Li}^{\mathbf{1}}$ and Wei Zhao ${ }^{2}$ \\ ${ }^{1}$ School of Information Science E Technology, East China Normal University, No. 500, Dong-Chuan Road, \\ Shanghai 200241, China \\ ${ }^{2}$ University of Macau, Avenue Padre Tomás Pereira, Taipa, Macau, China \\ Correspondence should be addressed to Ming Li, ming lihk@yahoo.com
}

Received 19 September 2009; Accepted 4 November 2009

Academic Editor: Carlo Cattani

Copyright (C) 2010 M. Li and W. Zhao. This is an open access article distributed under the Creative Commons Attribution License, which permits unrestricted use, distribution, and reproduction in any medium, provided the original work is properly cited.

This paper discusses the estimation of autocorrelation function (ACF) of fractional Gaussian noise (fGn) with long-range dependence (LRD). A variance bound of ACF estimation of one block of fGn with LRD for a given value of the Hurst parameter $(H)$ is given. The present bound provides a guideline to require the block size to guarantee that the variance of ACF estimation of one block of fGn with LRD for a given $H$ value does not exceed the predetermined variance bound regardless of the start point of the block. In addition, the present result implies that the error of ACF estimation of a block of fGn with LRD depends only on the number of data points within the sample and not on the actual sample length in time. For a given block size, the error is found to be larger for fGn with stronger LRD than that with weaker LRD.

\section{Introduction}

ACF analysis, or equivalently spectral analysis according to the Wiener-Khintchine theorem, plays a role in many areas of sciences and technologies (see, e.g., [1, 2]), such as structural engineering [3-7]. In engineering, ACF or its Fourier transform (power spectrum density function (PSD)) can only be estimated according to a given record length in measurement. Note that the random load simulated in a laboratory test may be generated based on a predetermined ACF or PSD; see, for example, [8-13]. Thus, the quality of ACF or PSD estimation has great impact on structure analysis and design.

The literature of error analysis (mainly, bias, and variance) of ACF/PSD estimation of an ordinary random process is quite rich; see, for example, [1, 2, 14-19]. By ordinary random processes, we mean that the ACF and PSD of a process are ordinary functions except the Dirac delta function that is the ACF of white noise. 
Note that processes with LRD or long-memory substantially differ from ordinary processes [20]. By LRD, we mean that the ACF of a process is nonsummable in the discrete case or nonintegrable in the continuous case [20]. Hence, its PSD should be considered in the sense of generalized function over the Schwartz space of test functions. FGn introduced in [21] is a widely used model of stationary fractal time series, which has found increasingly wide applications in many fields of sciences and technologies, ranging from hydrology to network traffic; see, for example, [22-45]. Note that the statistics of a zero mean Gaussian process are completely determined in terms of its ACF. Therefore, when using fGn-type load in structural engineering, the method to assure the quality of its ACF estimation is desired. In passing, we mention that, in the field of the Internet, ACF estimation of fGn-type teletraffic is utilized for detection of distributed denial-of-service flood attacks [32].

In the field, [46] discussed the statistical error of the structure function of Gaussian random fractals, and [47] studied the bias of the sample autocorrelations of fractional noise. This paper aims at providing a variance bound of the ACF estimation of one block of fGn.

An ACF is usually estimated on a block-by-block basis [1, 10, 13], where block size means the number of data points of a block of sample. Note that the ACF estimation of different blocks may be different, resulting in the estimation error caused by sectioning. The error resulted from sectioning can be reduced by the skill of averaging [1]. Different from conventional methods to reduce errors based on averaging, this research studies how to determine the size of one block according to a given degree of accuracy of ACF estimation of fGn with LRD.

Intuitively, if the size of one block is large enough, the ACF estimation will be independent of the start point for sectioning the block. Let $N$ be the block size of fGn with LRD. The aim of this paper is to provide a formula to calculate the variance bound of ACF estimation of fGn with LRD for a given $N$ and a given value of $H$.

The remaining article is organized as follows. Section 2 presents an error bound of ACF estimation of one block of fGn with LRD. Discussions are given in Section 3. Finally, Section 4 concludes the paper.

\section{Variance Bound of ACF Estimation of One Block of fGn with LRD}

\subsection{Preliminaries}

Let $B(t)$ be ordinary Brownian motion $(\mathrm{Bm})$ for $t \geq 0$ and $B(0)=0$ [48]. The stationary white noise can be taken as $B^{\prime}(t)$, which is the derivative of $B(t)$ in the domain of generalized functions. Let ${ }_{0} D_{t}^{-v}$ be the Riemann-Liouville integral operator $[49,50]$. Then,

$$
{ }_{0} D_{t}^{-v} B^{\prime}(t)=\frac{1}{\Gamma(v)} \int_{0}^{t}(t-u)^{v-1} d B(u)
$$

where $\Gamma$ is the Gamma function. Replacing $v$ with $H+1 / 2$ in (2.1) yields

$$
{ }_{0} D_{t}^{-(H+1 / 2)} B^{\prime}(t)=\frac{1}{\Gamma(H+1 / 2)} \int_{0}^{t}(t-u)^{H-1 / 2} d B(u) \triangleq B_{H}^{0}(t)
$$

In the above expression, $B_{H}^{0}(t)$ is termed the Riemann-Liouville fractional Brownian motion $(\mathrm{fBm})$ and $0<H<1$. This $\mathrm{fBm}$ is self-similar but does not have stationary increments. In 
passing, it is noted that the $\mathrm{fBm}$ described in the sense of the Riemann-Liouville fractional integral can be explained as the response of a fractional system, the impulse response of which is $t^{H-1 / 2} / \Gamma(H+1 / 2)$ under the excitation of white noise from a view of the theory of linear fractional systems discussed in [51,52].

Following Mandelbrot and van Ness [21], the fBm that is self-similar and has stationary increments is defined for $t>0$ by

$$
B_{H}(t)-B_{H}(0)=\frac{1}{\Gamma(H+1 / 2)}\left\{\begin{array}{c}
\int_{-\infty}^{0}\left[(t-u)^{H-0.5}-(-u)^{H-0.5}\right] d B(u) \\
+\int_{0}^{t}(t-u)^{H-0.5} d B(u)
\end{array}\right\},
$$

where $B_{H}(0)=b_{0}$ is the starting value at time 0 . If $b_{0}=0, B_{1 / 2}(t)=B(t)$. Hence, $\mathrm{fBm}$ generalizes $\mathrm{Bm}$. The $\mathrm{fBm}$ expressed by (2.3) is the fractional integral of $B(t)$ in the sense of Weyl (see $[49,50]$ for the details of the fractional Weyl integral operator).

FGn is the increment process of $\mathrm{fBm}$. It is stationary and self-affine with parameter $H$. Let $X(t)$ be $\mathrm{fGn}$ in the continuous case. Then, the ACF of $X(t)$ is given by

$$
r(\tau)=\frac{\sigma^{2} \varepsilon^{2 H-2}}{2}\left[\left(\frac{|\tau|}{\varepsilon}+1\right)^{2 H}+\left|\frac{|\tau|}{\varepsilon}-1\right|^{2 H}-2\left|\frac{\tau}{\varepsilon}\right|^{2 H}\right], \quad \tau \in \mathbb{R}
$$

where $0<H<1, \sigma^{2}=(H \pi)^{-1} \Gamma(1-2 H) \cos (H \pi)$ is the intensity of fGn, and $\varepsilon>0$ is used by regularizing $\mathrm{fBm}$ so that the regularized $\mathrm{fBm}$ is differentiable [21, pages 427-428]. The PSD of $X(t)$ is given by ( $\mathrm{Li}$ and $\mathrm{Lim}[53])$

$$
S(\omega)=\sigma^{2} \sin (H \pi) \Gamma(2 H+1)|\omega|^{1-2 H} .
$$

Letting $\varepsilon=1$ and replacing $\tau \in \mathbb{R}$ by $k \in \mathbb{Z}$ in (2.4) yields the ACF of the discrete fGn (dfGn):

$$
r(k)=\frac{\sigma^{2}}{2}\left[(|k|+1)^{2 H}+|| k|-1|^{2 H}-2|k|^{2 H}\right], \quad k \in \mathbb{Z} .
$$

Recall that a stationary Gaussian process with ACF $r(\tau)$ is of LRD if [20]

$$
\int_{0}^{\infty} r(\tau) d \tau=\infty
$$

otherwise it is of short-range dependence (SRD). Another definition of LRD is given as follows. For asymptotically large time scales, if

$$
r(\tau) \sim \tau^{-\beta}, \quad \beta \in(0,1) \text { as } \tau \longrightarrow \infty,
$$

then the process is of LRD. 
Note that the expression $0.5\left[(k+1)^{2 H}-2 k^{2 H}+(k-1)^{2 H}\right]$ described in (2.6) is the finite second-order difference of $0.5(k)^{2 H}$. Approximating it with the second-order differential of 0.5 $(k)^{2 H}$ yields

$$
0.5\left[(k+1)^{2 H}-2 k^{2 H}+(k-1)^{2 H}\right] \approx H(2 H-1)(k)^{2 H-2} .
$$

Expressing $\beta$ in (2.8) by the Hurst parameter $H$ gives $\beta=2-2 H$, or

$$
H=1-\frac{\beta}{2}
$$

The LRD condition expressed by $H$ therefore is $0.5<H<1$. The larger the $H$ value, the stronger the long-range persistence.

FGn contains three subclasses of time series. In the case of $0.5<H<1, r(\tau)$ is positive and finite for all $\tau$. It is monotonously decreasing but nonintegrable. In fact, from the ACF of dfGn described by (2.9), one immediately has $\sum_{0}^{\infty} k^{2 H-2}=\infty$. Thus, for $0.5<H<1$, the corresponding $\mathrm{fGn}$ is of LRD. For $H \in(0,0.5)$, the integral of $r(\tau)$ is zero. Hence, $\mathrm{fGn}$ is of SRD in this case. Moreover, $r(\tau)$ changes its sign and becomes negative for some $\tau$ proportional to $\varepsilon$ in the parameter domain [21, page 434]. FGn reduces to white noise when $H=0.5$.

Note that if $r(\tau)$ is sufficiently smooth on $(0, \infty)$ and if

$$
r(0)-r(\tau) \sim c|\tau|^{\alpha} \quad \text { for }|\tau| \longrightarrow 0,
$$

where $c$ is a constant, then one has the fractal dimension of $X(t)$ as

$$
D=2-\frac{\alpha}{2}
$$

see, for example, [54-57]. The local irregularity of the sample paths is measured by $\alpha$, which can be regarded as the fractal index of the process. Thus, the behaviour of $r(\tau)$ near the origin determines the local irregularity or the local self-similarity of the sample paths. The larger the $D$ value, the higher the local irregularity.

Now, in the case of $\varepsilon=1$, we apply the binomial series to $r(\tau)$. Then, one has

$$
r(0)-r(\tau) \sim c|\tau|^{2 H} \quad \text { for }|\tau| \longrightarrow 0
$$

Therefore, one immediately gets

$$
D=2-H
$$

Hence, $H$ measures both LRD and self-similarity of fGn. In other words, the local properties of fGn are reflected in the global ones as remarked by Mandelbrot [58, page 27].

Figures $1(\mathrm{a})$ and $1(\mathrm{~b})$ give the plots of the ACFs of fGn with LRD and SRD in the case of $\varepsilon=1$, respectively. 


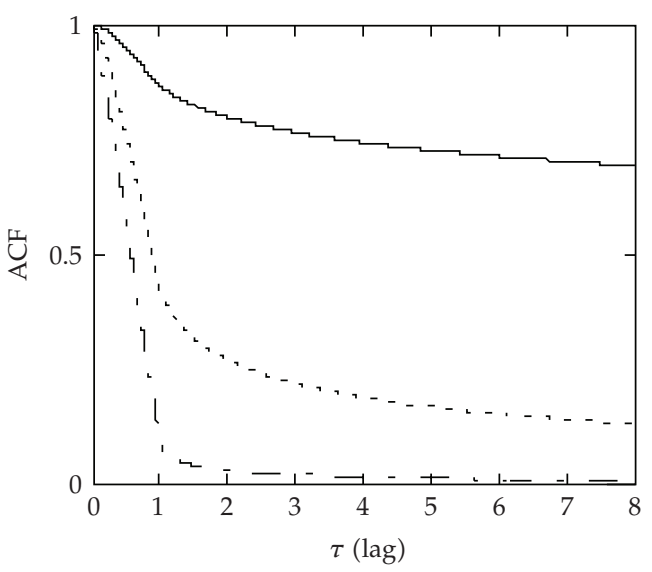

(a)

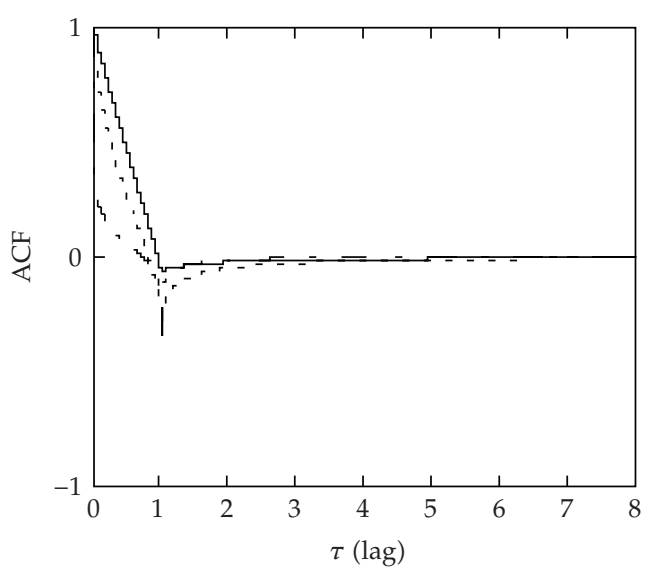

(b)

Figure 1: Plots of ACF of fractional Gaussian noise. (a) ACF of fGn with LRD. Solid line is for $H=0.95$, dot line is for $H=0.75$, and dadot line is for $H=0.55$. (b) ACF of fGn with SRD. Solid line is for $H=0.45$, dot line is for $H=0.25$, and dadot line is for $H=0.05$.

\subsection{Variance Bound}

In practical terms, the number of measured data points within a sample of $\mathrm{fGn}$ is finite. Let a positive integer $N$ be the number of data points of a measured sample of dfGn sequence $x(i)$. Then, the ACF of $x(i)$ is estimated by

$$
R(k)=\frac{1}{N} \sum_{i=1}^{N} x(i) x(i+k)
$$

Usually, for $l, m \in \mathbb{Z}_{+}$,

$$
\frac{1}{N} \sum_{i=m N}^{(m+1) N} x(i) x(i+k) \neq \frac{1}{N} \sum_{i=l N}^{(l+1) N} x(i) x(i+k)
$$

Therefore, $R(k)$ is a random variable.

Let $M^{2}(R)$ be the mean square error in terms of $R$. Denote $R(k)$ by $R(k ; H, N)$. The aim of the statistical error analysis in this research is to derive a relationship between $M^{2}(R)$ and $N$ as well as $H$ so as to establish a reference guideline for requiring $N$ under the conditions that the bound of $M^{2}(R)$ and the value of $H$ are given.

Theorem 2.1. Let $x(i)$ be dfGn series with LRD. Let $r(k)$ be the true ACF of $x(i)$. Let $N$ be the number of data points of a sample sequence. Let $R(k)$ be an estimate of $r(k)$. Let $\operatorname{Var}(R)$ be the variance of $R$. Then,

$$
\operatorname{Var}(R) \leq \frac{4|\Gamma(1-2 H)|^{2} \cos ^{2}(H \pi)(2 H-1)^{2}}{\pi^{2} N} \sum_{i=1}^{N}(i)^{4 H-4}
$$


Proof. Mathematically, $r(k)$ is computed over infinite interval $[1,2,59]$ :

$$
r(k)=E[x(i) x(i+k)]=\lim _{N \rightarrow \infty} \frac{1}{N} \sum_{i=0}^{N} x(i) x(i+k) .
$$

In practice, $r(k)$ can only be estimated with a finite sequence. Therefore,

$$
r(k) \approx R(k)=\frac{1}{N} \sum_{i=N_{0}}^{N_{0}+N} x(i) x(i+k)
$$

where $N_{0}$ is the start point.

Let $b^{2}(R)$ be the bias of $R$. Then, $M^{2}(R)=E\left[(r-R)^{2}\right]=\operatorname{Var}(R)+b^{2}(R)$. Since

$$
E[R(\tau)]=\frac{1}{N} \sum_{i=N_{0}}^{N_{0}+N} E[x(i) x(i+k)]=\frac{1}{N} \sum_{i=N_{0}}^{N_{0}+N} r(k)=r(k),
$$

$R(k)$ is the unbiased estimate of $r(k)$ and $M^{2}(R)=\operatorname{Var}(R)$ accordingly. We need to express $\operatorname{Var}(R)$ by the following proposition to prove the theorem.

Proposition 2.2. Let $x(i)$ be dfGn with LRD. Let $r(k)$ be the true ACF of $x(i)$. Let $N$ be the number of data points of a sample sequence. Let $R(k)$ be an estimate of $r(k)$. Let $\operatorname{Var}(R)$ be the variance of $R$. Suppose that $r(k)$ is monotonously decreasing and $r(k) \geq 0$. Then,

$$
\operatorname{Var}(R) \leq \frac{4}{N} \sum_{i=0}^{N} r^{2}(k)
$$

Proof. As $\operatorname{Var}(R)=E\left\{[R-E(R)]^{2}\right\}=E\left(R^{2}\right)-E^{2}(R)$, according to (P-3), one has

$$
\operatorname{Var}(R)=E\left(R^{2}\right)-r^{2}
$$

Expanding $E\left(R^{2}\right)$ yields

$$
\begin{aligned}
E\left(R^{2}\right) & =E\left\{\left[\frac{1}{N} \sum_{N_{0}}^{N_{0}+N} x(i) x(i+k)\right]^{2}\right\} \\
& =E\left[\frac{1}{N^{2}} \sum_{N_{0}}^{N_{0}+N} x\left(i_{1}\right) x\left(i_{1}+k\right) \sum_{N_{0}}^{N_{0}+N} x\left(i_{2}\right) x\left(i_{2}+k\right)\right] \\
& =E\left[\frac{1}{N^{2}} \sum_{N_{0}}^{N_{0}+N} \sum_{N_{0}}^{N_{0}+N} x\left(i_{1}\right) x\left(i_{2}\right) x\left(i_{1}+k\right) x\left(i_{2}+k\right)\right] \\
& =\frac{1}{N^{2}} \sum_{N_{0}}^{N_{0}+N} \sum_{N_{0}}^{N_{0}+N} E\left[x\left(i_{1}\right) x\left(i_{2}\right) x\left(i_{1}+k\right) x\left(i_{2}+k\right)\right] .
\end{aligned}
$$


Thus,

$$
\operatorname{Var}(R)=\frac{1}{N^{2}} \sum_{N_{0}}^{N_{0}+N} \sum_{N_{0}}^{N_{0}+N} E\left[x\left(i_{1}\right) x\left(i_{2}\right) x\left(i_{1}+k\right) x\left(i_{2}+k\right)\right]-r^{2}(k) .
$$

Let

$$
\begin{gathered}
X_{1}=x\left(n_{1}\right), \\
X_{2}=x\left(n_{2}\right), \\
X_{3}=x\left(n_{1}+k\right), \\
X_{4}=x\left(n_{2}+k\right) .
\end{gathered}
$$

Then,

$$
E\left[x\left(n_{1}\right) x\left(n_{2}\right) x\left(n_{1}+k\right) x\left(n_{2}+k\right)\right]=E\left(X_{1} X_{2} X_{3} X_{4}\right) .
$$

Since $x$ is Gaussian, random variables $X_{1}, X_{2}, X_{3}$, and $X_{4}$ have a joint-normal distribution and $E\left(X_{1} X_{2} X_{3} X_{4}\right)=m_{12} m_{34}+m_{13} m_{24}+m_{14} m_{23}$, where

$$
\begin{gathered}
m_{12}=E\left[x\left(n_{1}\right) x\left(n_{2}\right)\right]=r\left(n_{2}-n_{1}\right), \\
m_{13}=E\left[x\left(n_{1}\right) x\left(n_{1}+k\right)\right]=r(k), \\
m_{14}=E\left[x\left(n_{1}\right) x\left(n_{2}+k\right)\right]=r\left(n_{2}-n_{1}+k\right), \\
m_{23}=E\left[x\left(n_{2}\right) x\left(n_{1}+k\right)\right]=r\left(n_{1}-n_{2}+k\right), \\
m_{24}=E\left[x\left(n_{2}\right) x\left(n_{2}+k\right)\right]=r(k), \\
m_{34}=E\left[x\left(n_{1}\right) x\left(n_{2}+k\right)\right]=r\left(n_{2}-n_{1}\right) .
\end{gathered}
$$

Therefore,

$$
\begin{aligned}
& \frac{1}{N^{2}} \sum_{N_{0}}^{N_{0}+N} \sum_{N_{0}}^{N_{0}+N} E\left[x\left(i_{1}\right) x\left(i_{2}\right) x\left(i_{1}+k\right) x\left(i_{2}+k\right)\right] \\
& \quad=\frac{1}{N^{2}} \sum_{N_{0}}^{N_{0}+N} \sum_{N_{0}}^{N_{0}+N} E\left(X_{1} X_{2} X_{3} X_{4}\right) \\
& =\frac{1}{N^{2}} \sum_{N_{0}}^{N_{0}+N} \sum_{N_{0}}^{N_{0}+N}\left(m_{12} m_{34}+m_{13} m_{24}+m_{14} m_{23}\right)
\end{aligned}
$$




$$
\begin{aligned}
& =\frac{1}{N^{2}} \sum_{N_{0}}^{N_{0}+N} \sum_{N_{0}}^{N_{0}+N} r^{2}\left(i_{2}-i_{1}\right)+r^{2}(k)+r\left(i_{2}-i_{1}+k\right) r\left(i_{1}-i_{2}+k\right) \\
& =\frac{1}{N^{2}} \sum_{N_{0}}^{N_{0}+N} \sum_{N_{0}}^{N_{0}+N} r^{2}\left(i_{2}-i_{1}\right)+r\left(i_{2}-i_{1}+k\right) r\left(i_{1}-i_{2}+k\right)+r^{2}(k) .
\end{aligned}
$$

According to (P-6), the variance is expressed as

$$
\operatorname{Var}(R)=\frac{1}{N^{2}} \sum_{N_{0}}^{N_{0}+N} \sum_{N_{0}}^{N_{0}+N} r^{2}\left(i_{2}-i_{1}\right)+r\left(i_{2}-i_{1}+k\right) r\left(i_{1}-i_{2}+k\right)
$$

Replacing $\left(i_{2}-i_{1}\right)$ with $i$ in the above expression yields

$$
\operatorname{Var}(R)=\frac{1}{N^{2}} \sum_{i_{1}=N_{0}}^{N_{0}+N} \sum_{i=N_{0}}^{N_{0}-N_{1}+N} r^{2}(i)+r(i+k) r(-i+k)=\frac{1}{N^{2}} \sum_{i_{1}=N_{0}}^{N_{0}+N} \sum_{i=N_{0}}^{N_{0}-N_{1}+N} f(i)
$$

where $f(i)=r^{2}(i)+r(i+k) r(-i+k)$. Without losing generality, let $N_{0}=0$. Then, the above becomes

$$
\operatorname{Var}(R)=\frac{1}{N^{2}} \sum_{i=0}^{N}(N-i) f(i)+\frac{1}{N^{2}} \sum_{i=-N}^{0}(N+i) f(i) .
$$

Since ACF is an even function, the above expression is written by

$$
\begin{aligned}
\operatorname{Var}(R) & =\frac{2}{N^{2}} \sum_{i=0}^{N}(N-i) f(i)=\frac{2}{N^{2}} \sum_{i=0}^{N}(N-i)\left[r^{2}(i)+r(i+k) r(-i+k)\right] \\
& \leq \frac{2}{N} \sum_{i=0}^{N}\left|1-\frac{i}{N}\right|\left|r^{2}(i)+r(i+k) r(-i+k)\right| \\
& \leq \frac{2}{N} \sum_{i=0}^{N}\left|r^{2}(i)+r(i+k) r(-i+k)\right| \leq \frac{4}{N} \sum_{i=0}^{N} r^{2}(i) .
\end{aligned}
$$

Therefore, Proposition 2.2 holds.

Now, replacing $r(k)$ with (2.6) yields

$$
\operatorname{Var}(R) \leq \frac{4}{N} \sum_{i=0}^{N} r^{2}(i) \leq \frac{4}{N} \sum_{i=1}^{N} r^{2}(i) \leq \frac{\sigma^{4}}{N} \sum_{i=1}^{N}\left[(i+1)^{2 H}-2 i^{2 H}+(i-1)^{2 H}\right]^{2} .
$$




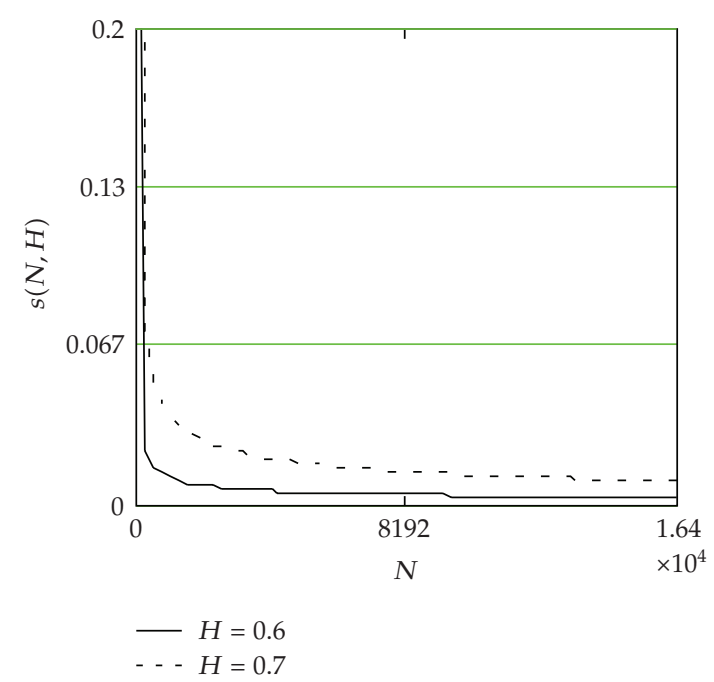

(a)

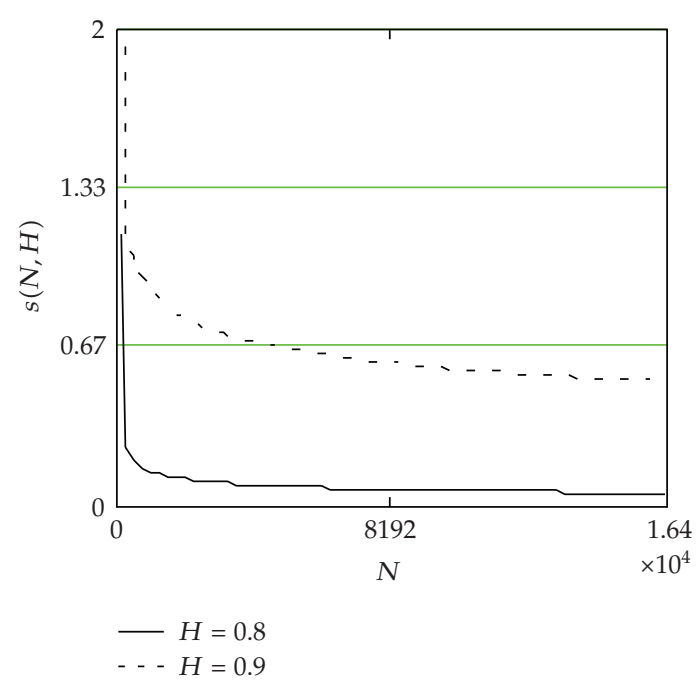

(b)

Figure 2: Error bound $s(N, H)$. (a) $H=0.60,0.70$. (b) $H=0.80,0.90$.

According to (2.9), replacing $\left[(i+1)^{2 H}-2 i^{2 H}+(i-1)^{2 H}\right]$ on the right hand of the above expression by $(i)^{2 H}$, we have

$$
\operatorname{Var}(R) \leq \frac{4|\Gamma(1-2 H)|^{2} \cos ^{2}(H \pi)(2 H-1)^{2}}{\pi^{2} N} \sum_{i=1}^{N}(i)^{4 H-4}
$$

Theorem results.

The above formula represents an upper bound of $\operatorname{Var}(R)$. Denote by $s(N, H)$ the bound of standard deviation. Then,

$$
s(N, H)=\frac{2 \Gamma(1-2 H) \cos (H \pi)|2 H-1|}{\pi} \sqrt{\frac{1}{N} \sum_{i=1}^{N}(i)^{4 H-4}} .
$$

We illustrate $s(N, H)$ in terms of $N$ by Figure 2 for $H=0.60,0.70,0.80$, and 0.90 .

From Figure 2, we see that $s\left(N, H_{1}\right)>s\left(N, H_{2}\right)$ for $H_{1}>H_{2}$, meaning that the error of ACF estimation of $\mathrm{fGn}$ is larger with stronger LRD than that with weaker LRD.

\section{Discussions}

\subsection{To Avoid Misleading Result of ACF Estimation}

Recall that processes with LRD substantiality differ from those with SRD [20]. Therefore, possible SRD signs of an ACF estimate of an fGn series that is of LRD may be taken as a misleading result of ACF estimation. 


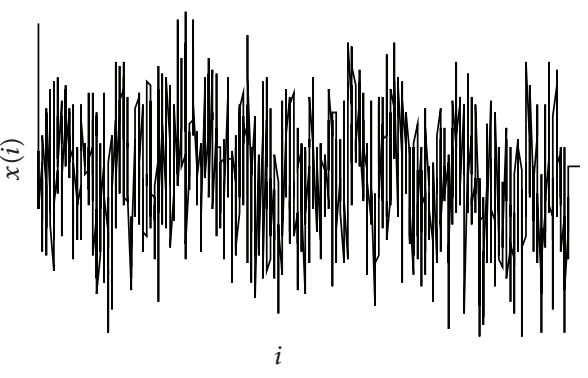

(a)

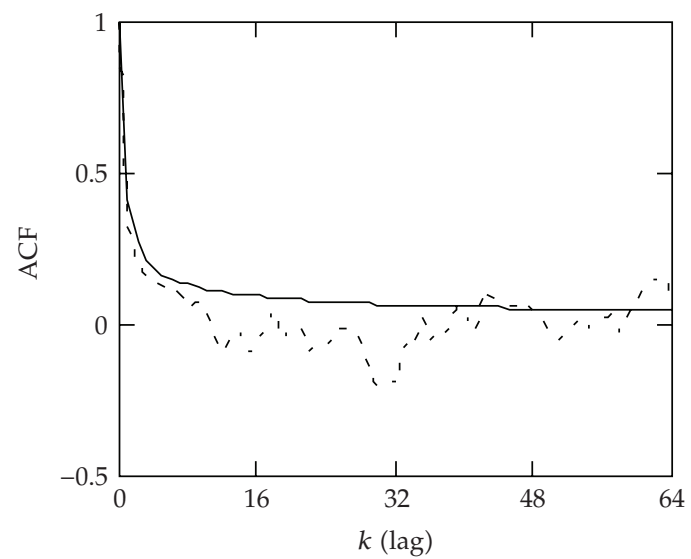

(c)

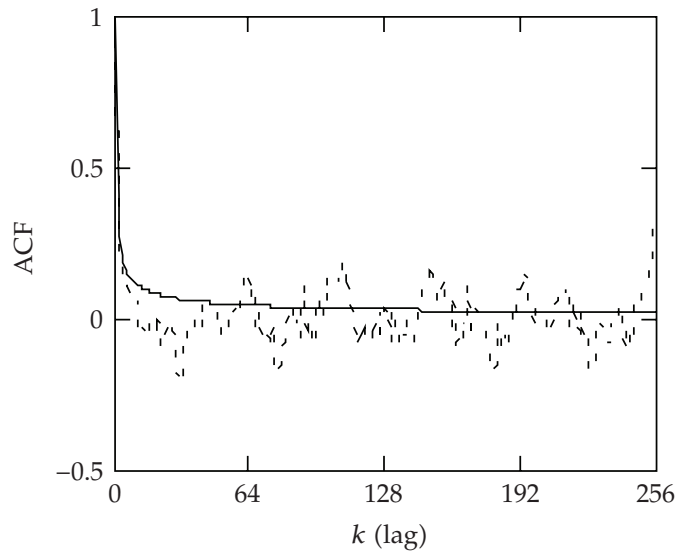

(b)

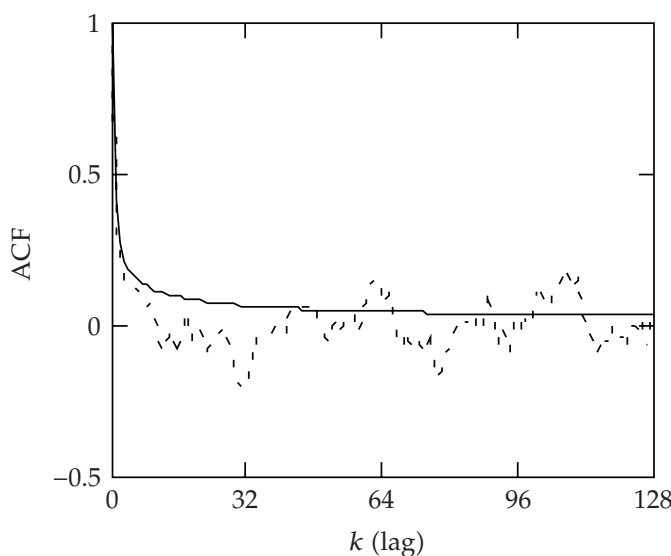

(d)

Figure 3: Case study. Solid line: theoretic ACF of fGn. Dot line: ACF estimate. (a) FGn with $H=0.75$. (b) AFC estimate for $N=256$. (c) The first 64 points of Figure 3(b). (d) The first 128 points of Figure 3(b).

Suppose that we have a block of fGn with $H=0.75$. Hence, this series is of LRD. Figure 3(a) shows an fGn series with $H=0.75$, which is synthesized with the method given in [60].

Assume the block size $N=256$. Then, we have $\operatorname{Var}(R) \leq 0.015$ according to Theorem 2.1. The dotted line in Figure 3(b) indicates its ACF estimation with $N=256$ and the solid line in Figure 3(b) shows the theoretical ACF of fGn with $H=0.75$. We note that the error regarding the ACF estimate reflected by the dotted line in Figure 3(b) is severe because many points of the dotted line are negative. Thus, it may probably confuse the property of the positive correlation (i.e., LRD) of the data being processed. Consequently, by the dotted line in Figure 3(b), one might likely be misled to take the data being processed (Figure 3(a)) as SRD. Figures 3(c) and 3(d) show the first 64 and 128 points of Figure 3(b), respectively. They again show the possible confusions caused by severe estimation error.

Now we increase the block size such that $N=2048$. Then, one has $\operatorname{Var}(R) \leq 2.5 \times$ $10^{-3}$ according to (2.17). In this case, the ACF estimation is indicated by the dotted line in Figure 4(a). Comparing Figure 4(a) to Figure 3(b), we see that the error of ACF estimation is 


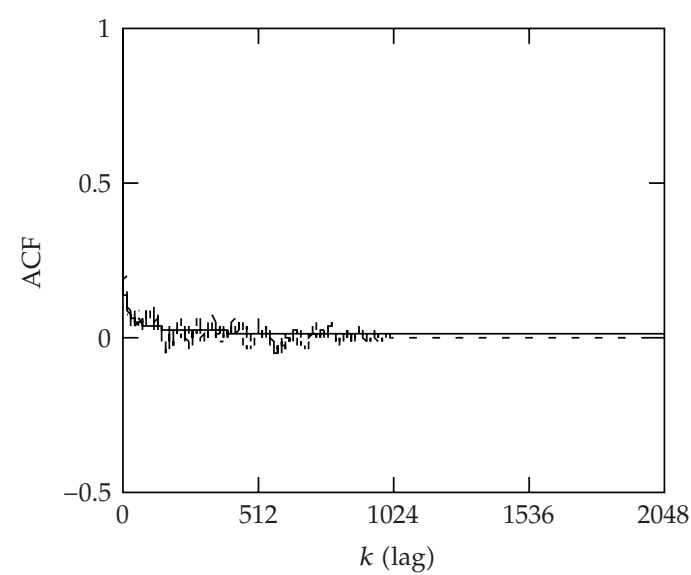

(a)

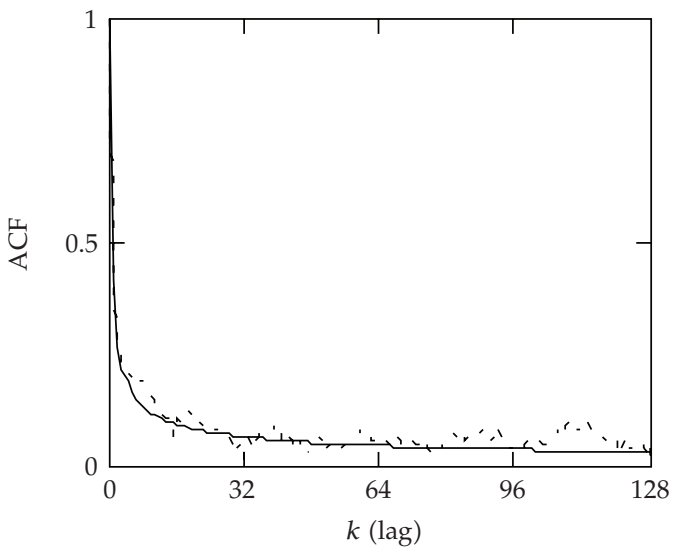

(c)

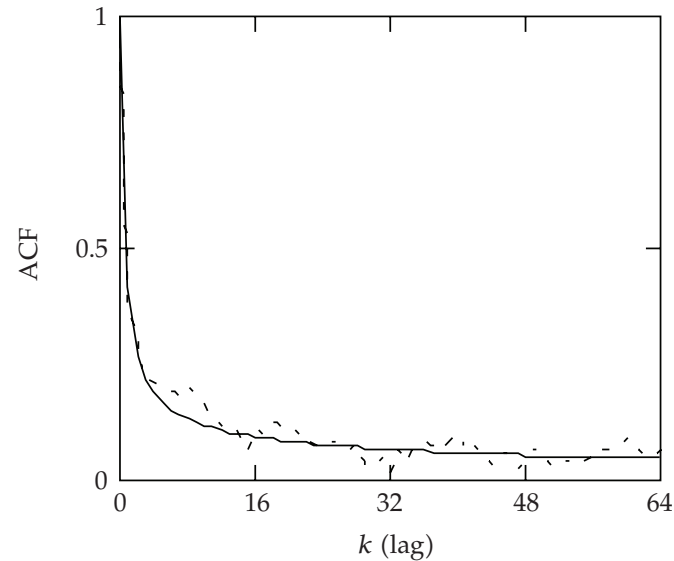

(b)

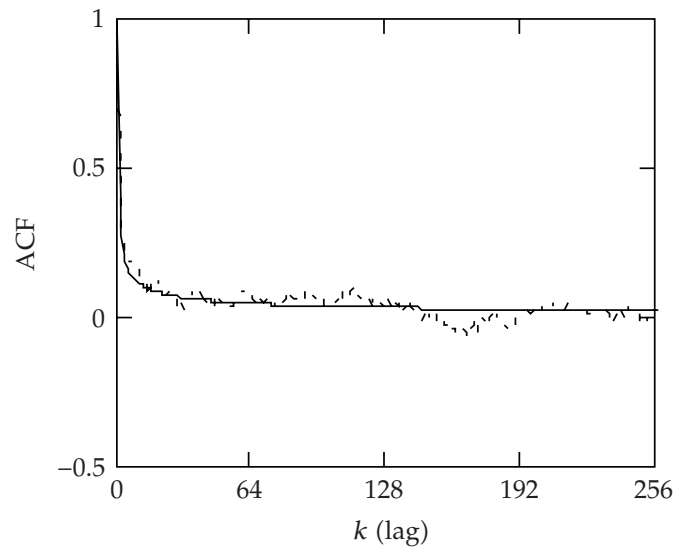

(d)

Figure 4: Solid line: theoretic ACF of FGN. Dot line: ACF estimate. (a) ACF estimate of fGn with $H=0.75$ for $N=$ 2048. (b) The first 64 points of Figure 4(b). (c) The first 128 points of Figure 4(b). (d) The first 256 points of Figure 4(b).

considerably reduced when $N$ increases to 2048 because most data points of the ACF estimate are positive. Figures 4(b), 4(c), and 4(d) give the plots of the first 64, 128, and 256 points of Figure 4(a), respectively. They evidently interpret the improvement of the quality of ACF estimation of one block of fGn with LRD by increasing the block size.

From the above, one sees that the accuracy of ACF estimate of fGn with LRD can be increased if the block size increases. Therefore, in addition to the direct way to increase the record length, increasing the sampling rate in measurement of fGn to be processed may yet be a way to increase the accuracy of the ACF estimation in the case that the block size is given.

\subsection{One Block Estimation}

The previous discussions regarding ACF estimation of fGn with LRD do not relate to averaging. In fact, once the block size $N$ is such that it meets the required accuracy according to Theorem 2.1, the ACF estimation is independent of the start point of the block. That is, for 
any $N_{0} \in \mathbb{Z}_{+},(1 / N) \sum_{i=N_{0}}^{N_{0}+N} x(i) x(i+k)$ yields an ACF estimate, the error of which is bounded based on Theorem 2.1. Further, we note that the discussed ACF estimation does not relate to sectioning. As a matter of fact, for each $m \in \mathbb{Z}_{+},(1 / N) \sum_{i=m N}^{(m+1) N} x(i) x(i+k)$ yields an ACF estimate, the error of which is bounded by (2.17).

\subsection{Remarks}

In the field of fractional order signal processing (see, e.g., [61]), [62] recently introduced a method to obtain a reliable estimation of $H$ based on fractional Fourier transform for processing very long experimental time series locally. It is worth noting that the present error bound in this paper may yet be an explanation why the reliable estimation of $H$ discussed in [62] requires long series.

Finally, we note that the ACF estimate expressed by (2.15) is biased one. However, that does not matter because the present variance bound relates to the fluctuation of the ACF estimate regardless of whether it is biased or not.

\section{Conclusions}

We have established an error bound of ACF estimation of one block of fGn with LRD. It has been shown that the error does not depend on the absolute length of the sample but only relies on the number of data points, that is, the block size $N$, of the sample. The error of an ACF estimate of fGn with stronger LRD is larger than that with weaker LRD for a given $N$. The discussed ACF estimation is not related to averaging. The accuracy of an ACF estimate of a block of fGn with LRD can be guaranteed once the block size is selected according to (2.17) without the relation to sectioning.

\section{Acknowledgment}

This work was supported in part by the National Natural Science Foundation of China under the project Grant nos. 60573125, 60873264, and 60873102.

\section{References}

[1] S. K. Mitra and J. F. Kaiser, Handbook for Digital Signal Processing, John Wiley \& Sons, New York, NY, USA, 1993.

[2] J. S. Bendat and A. G. Piersol, Random Data: Analysis and Measurement Procedure, John Wiley \& Sons, New York, NY, USA, 3rd edition, 2000.

[3] I. Elishakoff and R. H. Lyon, Random Vibration Status and Recent Developments, vol. 14 of Studies in Applied Mechanics, Elsevier, New York, NY, USA, 1986.

[4] A. Preumont, Random Vibration and Spectral Analysis, vol. 33 of Studies in Applied Mechanics, Kluwer, London, UK, 1993.

[5] J. J. Jensen and M. Dogliani, "Wave-induced ship hull vibrations in stochastic seaways," Marine Structures, vol. 9, no. 3-4, pp. 353-387, 1996.

[6] X.-K. Gu, J.-J. Hu, and J.-W. Shen, "The frequency characteristics of ship vertical wave bending moments," Shipbuilding of China, vol. 41, no. 1, pp. 31-38, 2000 (Chinese).

[7] D. Benasciutti and R. Tovo, "Spectral methods for lifetime prediction under wide-band stationary random processes," International Journal of Fatigue, vol. 27, no. 8, pp. 867-877, 2005.

[8] Technical Software Consultants Limited, "FLAPS: Fatigue Laboratory Applications Package, Version 01:03," Instron 2165035, UK. 
[9] M. Li, "An optimal controller of an irregular wave maker," Applied Mathematical Modelling, vol. 29, no. 1 , pp. 55-63, 2005.

[10] M. Li, "An iteration method to adjusting random loading for a laboratory fatigue test," International Journal of Fatigue, vol. 27, no. 7, pp. 783-789, 2005.

[11] M. Li, "Experimental stability analysis of a test system for doing fatigue tests under random loading," Journal of Testing and Evaluation, vol. 34, no. 4, pp. 364-367, 2006.

[12] M. Li, Y.-S. Wu, B.-H. Xu, W. Jia, and W. Zhao, "An on-line correction technique of random loading with a real-time signal processor for a laboratory fatigue test," Journal of Testing and Evaluation, vol. 28, no. 5, pp. 409-414, 2000.

[13] M. Li, B.-H. Xu, and Y.-S. Wu, "An $\mathrm{H}_{2}$-optimal control of random loading for a laboratory fatigue test," Journal of Testing and Evaluation, vol. 26, no. 6, pp. 619-625, 1998.

[14] F. H. C. Marriott and J. A. Pope, "Bias in the estimation of autocorrelations," Biometrika, vol. 41, pp. 390-402, 1954.

[15] E. Jakeman, E. R. Pike, and S. Swain, "Statistical accuracy in the digital autocorrelation of photon counting fluctuations," Journal of Physics A, vol. 4, no. 4, pp. 517-534, 1971.

[16] J. Pickands III, "Spectral estimation with random truncation," The Annals of Mathematical Statistics, vol. 41, no. 1, pp. 44-58, 1970.

[17] U. Grenander, "Bandwidth and variance in estimation of the spectrum," Journal of the Royal Statistical Society Series B, vol. 20, no. 1, pp. 152-157, 1958.

[18] D. J. Thomson, "Spectrum estimation and harmonic analysis," Proceedings of the IEEE, vol. 70, no. 9, pp. 1055-1096, 1982.

[19] M. Li, "A method for requiring block size for spectrum measurement of ocean surface waves," IEEE Transactions on Instrumentation and Measurement, vol. 55, no. 6, pp. 2207-2215, 2006.

[20] J. Beran, Statistics for Long-Memory Processes, vol. 61 of Monographs on Statistics and Applied Probability, Chapman \& Hall, New York, NY, USA, 1994.

[21] B. B. Mandelbrot and J. W. van Ness, "Fractional Brownian motions, fractional noises and applications," SIAM Review, vol. 10, no. 4, pp. 422-437, 1968.

[22] H. E. Hurst, "Long-term storage capacity of reservoirs," Transactions of American Society of Civil Engineers, vol. 116, pp. 770-808, 1951.

[23] B. B. Mandelbrot and J. R. Wallis, “Noah, Joseph and operational hydrology," Water Resources Research, vol. 4, no. 5, pp. 909-918, 1968.

[24] S. M. Barbosa, M. J. Fernandes, and M. E. Silva, "Long-range dependence in North Atlantic sea level," Physica A, vol. 371, no. 2, pp. 725-731, 2006.

[25] A. J. Lawrance and N. T. Kottegoda, "Stochastic modelling of riverflow time series," Journal of the Royal Statistical Society-Series A, vol. 140, no. 1, pp. 1-47, 1977.

[26] M. Radziejewski and Z. W. Kundzewicz, "Fractal analysis of flow of the river Warta," Journal of Hydrology, vol. 200, no. 1-4, pp. 280-294, 1997.

[27] B. B. Mandelbrot, Gaussian Self-Affinity and Fractals, Selected Works of Benoit B. Mandelbrot, Springer, New York, NY, USA, 2002.

[28] W. Stallings, High-Speed Networks TCP/IP and ATM Design Principles, chapter 8, Prentice-Hall, Upper Saddle River, NJ, USA, 1998.

[29] M. Li, "An approach to reliably identifying signs of DDOS flood attacks based on LRD traffic pattern recognition," Computers and Security, vol. 23, no. 7, pp. 549-558, 2004.

[30] B. B. Mandelbrot, Gaussian Self-Affinity and Fractals, Selected Works of Benoit B. Mandelbrot, Springer, New York, NY, USA, 2002.

[31] J. Levy-Vehel, E. Lutton, and C. Tricot, Eds., Fractals in Engineering, Springer, Berlin, Germany, 1997.

[32] M. Li and W. Zhao, "Detection of variations of local irregularity of traffic under DDOS flood attack," Mathematical Problems in Engineering, vol. 2008, Article ID 475878, 11 pages, 2008.

[33] M. Li, "Modeling autocorrelation functions of long-range dependent teletraffic series based on optimal approximation in Hilbert space-A further study," Applied Mathematical Modelling, vol. 31, no. 3, pp. 625-631, 2007.

[34] M. Li, "Change trend of averaged Hurst parameter of traffic under DDOS flood attacks," Computers E Security, vol. 25, no. 3, pp. 213-220, 2006.

[35] C. Cattani, "Shannon wavelets theory," Mathematical Problems in Engineering, vol. 2008, Article ID 164808, 24 pages, 2008.

[36] E. G. Bakhoum and C. Toma, "Relativistic short range phenomena and space-time aspects of pulse measurements," Mathematical Problems in Engineering, vol. 2008, Article ID 410156, 20 pages, 2008. 
[37] C. Cattani, "Harmonic wavelet analysis of a localized fractal," International Journal of Engineering and Interdisciplinary Mathematics, vol. 1, no. 1, pp. 35-44, 2009.

[38] P. Abry, P. Borgnat, F. Ricciato, A. Scherrer, and D. Veitch, "Revisiting an old friend: on the observability of the relation between long range dependence and heavy tail," Telecommunication Systems, 2009.

[39] M. Li, W.-S. Chen, and L. Han, "Correlation matching method of the weak stationarity test of LRD traffic," Telecommunication Systems, 2009.

[40] L. Janowski and P. Owezarski, "Assessing the accuracy of using aggregated traffic traces in network engineering," Telecommunication Systems, 2009.

[41] C. Li and W. Zhao, "Stochastic performance analysis of non-feedforward networks," Telecommunication Systems, 2009.

[42] M. Li, "Fractal time series-a tutorial," Mathematical Problems in Engineering, vol. 2010, Article ID 157264, 26 pages, 2010.

[43] M. Li and W. Zhao, "Representation of a stochastic traffic bound," IEEE Transactions on Parallel $\mathcal{E}$ Distributed Systems, 2009, http:/ / doi.ieeecomputersociety.org/10.1109/TPDS.2009.162.

[44] K.-L. Tian and M. Li, "A reliable anomaly detector against low-rate DDOS attack," International Journal of Electronics and Computers, vol. 1, no. 1, pp. 1-6, 2009.

[45] C. Cattani, "Harmonic wavelet approximation of random, fractal and high frequency signals," Telecommunication Systems. In press.

[46] E. Jakeman and D. L. Jordan, "Statistical accuracy of measurements on Gaussian random fractals," Journal of Physics D, vol. 23, no. 4, pp. 397-405, 1990.

[47] P. Newbold and C. Agiakloglou, "Bias in the sample autocorrelations of fractional noise," Biometrica, vol. 30, no. 3, pp. 698-702, 1990.

[48] T. Hida, Brownian Motion, vol. 11 of Applications of Mathematics, Springer, New York, NY, USA, 1980.

[49] A. A. Kilbas, H. M. Srivastava, and J. J. Trujillo, Theory and Applications of Fractional Differential Equations, vol. 204 of North-Holland Mathematics Studies, Elsevier Science, Amsterdam, The Netherlands, 2006.

[50] K. S. Miller and B. Ross, An Introduction to the Fractional Calculus and Fractional Differential Equations, A Wiley-Interscience Publication, John Wiley \& Sons, New York, NY, USA, 1993.

[51] M. D. Ortigueira, "Introduction to fractional linear systems, part I: continuous-time systems," IEE Proceedings: Vision, Image and Signal Processing, vol. 147, no. 1, pp. 62-70, 2000.

[52] M. D. Ortigueira and A. G. Batista, "A fractional linear system view of the fractional brownian motion," Nonlinear Dynamics, vol. 38, no. 1-4, pp. 295-303, 2004.

[53] M. Li and S. C. Lim, "A rigorous derivation of power spectrum of fractional Gaussian noise," Fluctuation and Noise Letters, vol. 6, no. 4, pp. C33-C36, 2006.

[54] R. J. Adler, The Geometry of Random Fields, Wiley Series in Probability and Mathematical Statistic, John Wiley \& Sons, New York, NY, USA, 1981.

[55] P. Hall and R. Roy, "On the relationship between fractal dimension and fractal index for stationary stochastic processes," The Annals of Applied Probability, vol. 4, no. 1, pp. 241-253, 1994.

[56] G. Chan, P. Hall, and D. S. Poskitt, "Periodogram-based estimators of fractal properties," The Annals of Statistics, vol. 23, no. 5, pp. 1684-1711, 1995.

[57] J. T. Kent and A. T. A. Wood, "Estimating the fractal dimension of a locally self-similar gaussian process by using increments," Journal of the Royal Statistical Society Series B, vol. 59, no. 3, pp. 679-699, 1997.

[58] B. B. Mandelbrot, The Fractal Geometry of Nature, W. H. Freeman, New York, NY, USA, 1982.

[59] G. A. Korn and T. M. Korn, Mathematical Handbook for Scientists and Engineers, McGraw-Hill, New York, NY, USA, 1961.

[60] M. Li and C.-H. Chi, "A correlation-based computational model for synthesizing long-range dependent data," Journal of the Franklin Institute, vol. 340, no. 6-7, pp. 503-514, 2004.

[61] Y. Chen, R. Sun, and A. Zhou, "An overview of fractional order signal processing (FOSP) techniques," in Proceedings of the ASME International Design Engineering Technical Conferences $\mathcal{E}$ Computers and Information in Engineering Conference (IDETC/CIE '07), vol. 5, pp. 1205-1222, Las Vegas, Nev, USA, September 2007, DETC2007-34228.

[62] Y.-Q. Chen, R. Sun, and A. Zhou, "An improved Hurst parameter estimator based on fractional Fourier transform," Telecommunication Systems, 2009. 


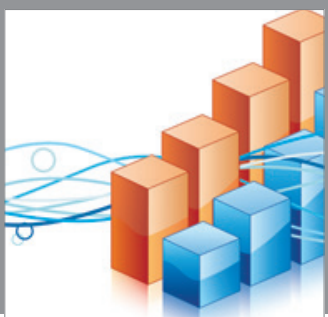

Advances in

Operations Research

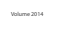

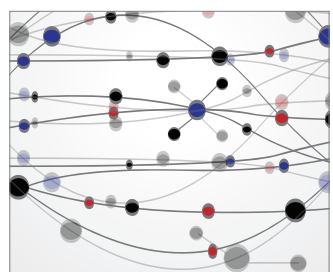

\section{The Scientific} World Journal
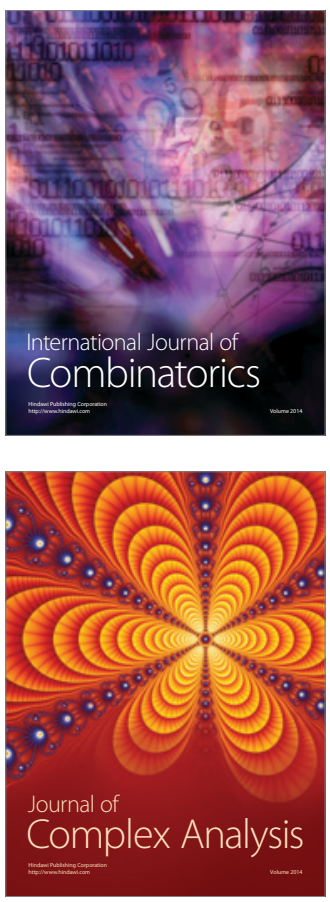

International Journal of

Mathematics and

Mathematical

Sciences
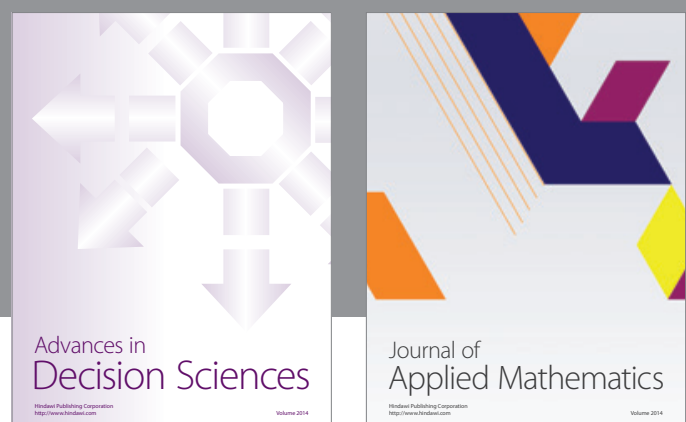

Journal of

Applied Mathematics
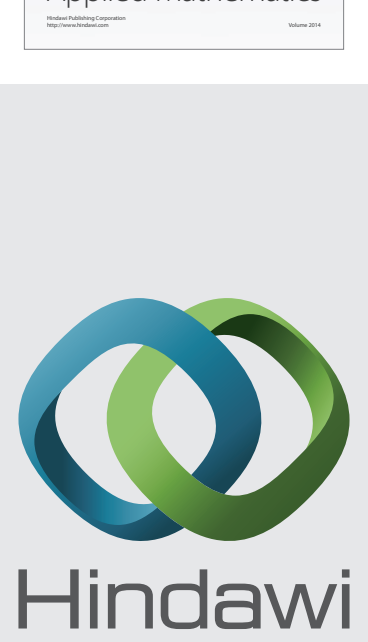

Submit your manuscripts at http://www.hindawi.com
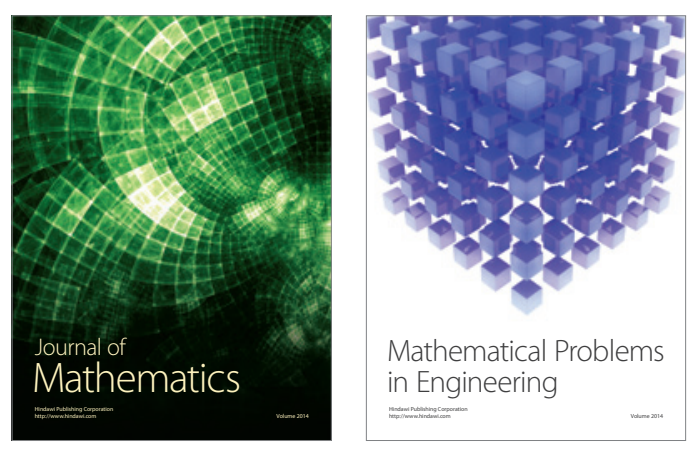

Mathematical Problems in Engineering
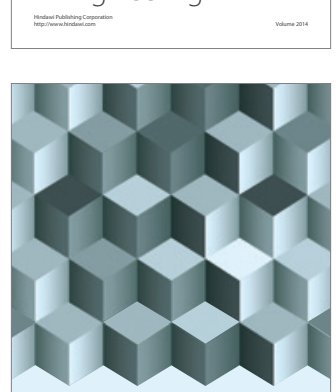

Journal of

Function Spaces
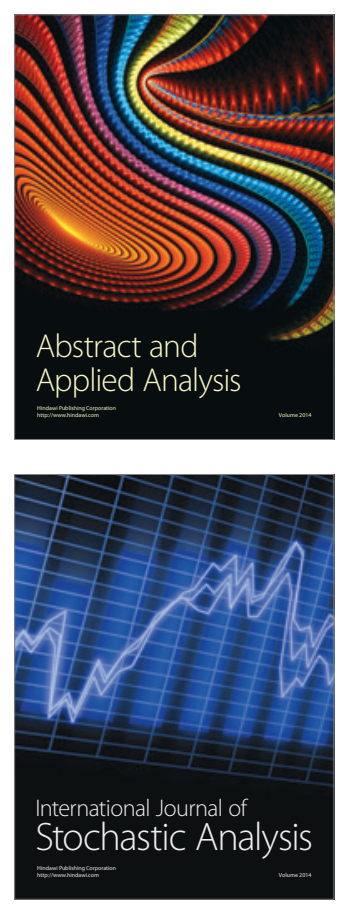

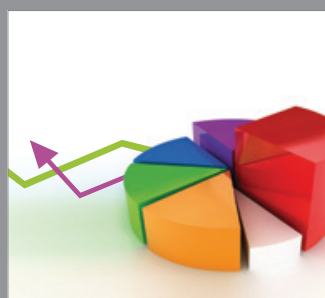

ournal of

Probability and Statistics

Promensencen
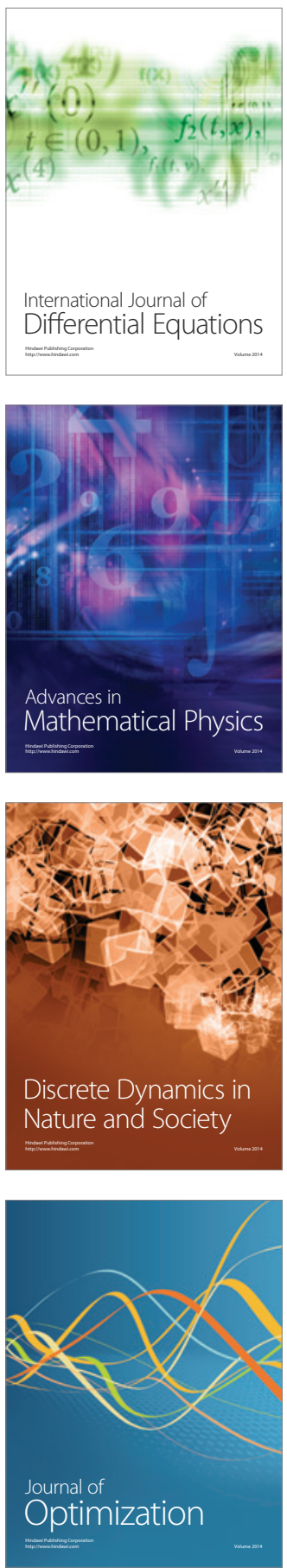